\title{
Sperm DNA fragmentation valued by SCSA and its correlation with conventional sperm parameters in male partner of recurrent spontaneous abortion couple
}

\author{
Minmin Yuan ${ }^{1}$, Liqing Huang ${ }^{2}$, Wing Ting Leung ${ }^{3}$, Mingyan Wang ${ }^{1}$, Yi Meng ${ }^{1}$, Zengshu Huang ${ }^{3}$, \\ Xinyao Pan ${ }^{3}$, Jing Zhou ${ }^{3}$, Chuyu Li ${ }^{3}$, Yizhen Sima ${ }^{3}$, Lan Wang ${ }^{3}$, Yanzhi Zhang ${ }^{3}$, Chunmei Ying ${ }^{1, *}$, \\ Ling Wang ${ }^{3,4,5}, *$ \\ ${ }^{1}$ Laboratory for Clinical Laboratory Testing, Hospital of Obstetrics and Gynecology, Fudan University Shanghai Medical \\ College, Shanghai, China; \\ ${ }^{2}$ Department of Statistics and Psychology, College of Letters and Science, University of California Davis, Davis, California, USA; \\ ${ }^{3}$ Laboratory for Reproductive Immunology, Hospital \& Institute of Obstetrics and Gynecology, Shanghai Medical College, Fudan \\ University, Shanghai, China; \\ ${ }^{4}$ The Academy of Integrative Medicine, Fudan University, Shanghai, China; \\ ${ }^{5}$ Shanghai Key Laboratory of Female Reproductive Endocrine-related Diseases, Shanghai, China.
}

\begin{abstract}
Summary The objective of this study is to evaluate the predictive value of sperm DNA fragmentation Index (DFI) in unexplained recurrent spontaneous abortion (RSA) and to investigate its correlation with conventional sperm parameters. Besides, we aimed to reveal the necessity of establishing a DFI clinical threshold of each laboratory for the prognostic diagnosis of RSA and establish our own DFI threshold. Semen samples were collected from male partners of RSA patients $(n=139)$ and healthy recent fathers (control, $n=200)$. DFI was tested using SCSA and conventional semen analysis was performed using an automatic semen analyzer. The DFI value and distribution were compared between the two groups using corresponding statistical software. The diagnostic threshold value was established by ROC curve. The correlation between DFI and the conventional semen parameters of the 139 cases was further analyzed using Student's $t$ test and Mann-Whitney $U$ test. Our result showed that DFI was significantly higher in RSA patients compared with normal donor controls. We established our own DFI threshold at $\mathbf{1 3 . 5 9 \%}$. There was only a weak partial correlation between DFI values and conventional sperm analysis parameters. Our present study suggested that DFI might be used as a valuable predictor for RSA independent of conventional sperm parameters. Additionally, we recommend that each laboratory should establish its own clinical DFI threshold for more precise prediction of RSA and we recommend that sperm DNA fragmentation test should be included in complete sperm quality assessment in addition to conventional semen analysis for RSA male partners.
\end{abstract}

Keywords: Recurrent spontaneous abortion (RSA), sperm DNA fragmentation (SDF), DNA fragmentation index (DFI), Sperm Chromatin Structure Assay (SCSA), high DNA stainability (HDS), semen analysis

Released online in J-STAGE as advance publication April 11, 2019.

*Address correspondence to:

Dr. Ling Wang, Obstetrics \& Gynecology Hospital of Fudan University, 419 Fangxie Road, Shanghai 200011, China.

E-mail: Dr.wangling@fudan.edu.cn

Dr. Chunmei Ying, Obstetrics \& Gynecology Hospital of Fudan University, 419 Fangxie Road, Shanghai 200011, China.

E-mail: ycmzh2012@163.com

\section{Introduction}

Recurrent spontaneous abortion (RSA) is a pregnancy complication whose etiology is still unclear. There have been many controversies regarding its diagnosis and treatment, but there is a general agreement that the incidence of RSA involves combined pathogenic factors from both males and females of the infertile 
couples. For decades, the initial diagnostic investigation of male fertility relies only on conventional semen analysis, which mainly includes sperm count, vitality, motility, and morphology according to World Health Organization (WHO) manual. No doubt that these parameters are closely relevant and important for the diagnosis of subfertility, which might be the cause of RSA. However, these parameters addressed only a few aspects of sperm quality and function, while in fact, an estimated $15-40 \%$ of males with normal conventional semen analysis results are nonetheless associated with infertility (1-3), or still suffer from repeated abortions. Thus, new tests for RSA male partners would be clinically useful.

In the past few decades, the role of sperm DNA integrity on fertility, embryo development, embryo quality, implantation and pregnancy has gained a lot of attention. As reported, fertilization of oocytes with spermatozoa that have damaged DNA could potentially lead to reduced fertilization rates, poor embryo quality, as well as higher rates of spontaneous miscarriage $(4,5)$. Also, the correlation between sperm DNA fragmentation (SDF) and recurrent pregnancy loss has been illustrated by many studies. Several studies have demonstrated a significantly higher DNA fragmentation index (DFI) in couples with RSA than in controls (68 ). However, although there are already considerable data supporting the clinical use of SDF tests, there are controversies regarding the exact value of DNA integrity in diagnostic and prognostic evaluation of RSA patients because some studies found no obvious differences in SDF level between RSA patients and healthy recent fathers (9). Some studies even denied that DNA fragmentation is one of the possible causes of RSA $(9,10)$. Thus, so far in China, only a few laboratories have implemented sperm chromatin integrity testing and adopted SDF as a routine scanning item to evaluate male's fertile capability. Therefore, more studies verifying the value of SDF testing in RSA couples are needed.

Despite some opposition, SDF has been proposed as a useful indicator for demonstrating the underlying causes of RSA and adopted as a scanning item in a few laboratories for male infertility, low assisted reproduction technology (ART) pregnancy rate, recurrent miscarriage and even severe adverse outcomes of the offspring such as cancer or neurological disorders (11-13). A variety of SDF detecting assays have been developed in the past few decades (14-16) but are limited in widespread use due to the inconsistent DFI threshold among different assays, the lack of standardized operation procedures and the lack of quality control among laboratories (17). Sperm chromatin structure assay (SCSA), firstly described by Evenson and his coworkers in 1980 (18) and improved later $(19,20)$, has been recommended as a relatively independent and reliable assay in China because of its better sensitivity, stability and accuracy. DFI clinical threshold values using SCSA with regard to fertility have been proposed and established in some laboratories (20). However, the DFI thresholds reported are inconsistent and conflicted (8). To our knowledge, for the laboratories in China that have implemented SDF testing for male fertility assessment, most of them just adopt the DFI cutoff value offered by the commercial kit producer which is usually $15 \%$. We hold the opinion that to use SDF testing more precisely in clinical practice, it is essential to establish a reliable clinical threshold value for each laboratory, which may reduce possible diagnostic error.

We expect to better illustrate these problems through our present study. Our work was comprised of the following parts. Firstly, we intended to reveal the clinical significance of using SDF test as a tool in evaluation of male fertility in RSA couples. Secondly, we aimed to investigate and establish the DFI clinical cutoff value of our laboratory, hoping to be helpful in establishment of a reference value for RSA male patients in Chinese Han population. Lastly, we further analyzed the correlation between DFI values and conventional semen analysis parameters to investigate the possible value of its future integration into routine clinical practice.

\section{Materials and Methods}

\subsection{Patients and data collection}

Patient data collection was approved by the Ethics Committees of Obstetrics \& Gynecology Hospital affiliated to Fudan University. A total of 139 RSA couples were enrolled in this study. Written informed consent was obtained from all cases. The 139 RSA couples were collected from the reproductive immunology clinic and recurrent miscarriage specialist clinic from October 2017 to March 2018. Patients inclusion criteria included a history of at least two unexplained first trimester recurrent spontaneous abortion and all the known female causes of recurrent pregnant loss were ruled out, and only idiopathic recurrent miscarriage cases were recruited. In brief, the female partner of couples enrolled was physically healthy with regular menstrual cycles, normal in hormonal profiles, and anatomically normal in uterus. Other causes of RSA like antiphospholipid syndrome or thrombophilia, metabolic disorders and genital infections were also screened and ruled out. Only male partner of each of these females was included in the following study. Both males and females of the couples had normal karyotype with no family history of physical diseases. Patients with other confounding factors such as varicocele, antioxidant intake, bad habits like excessive drinking and smoking, which may affect DNA damage levels were ruled out. The age of enrolled patients all ranged from 20 to 40 years old and 
were age matched with normal controls.

\subsection{Sample Collection and analysis}

The 139 male's fresh semen samples were obtained by masturbation at our laboratory in a sterile plastic container after 3-7 days of sexual abstinence. After liquefaction at room temperature, conventional semen analysis was performed with Weili automatic semen analyzer 9000 following WHO guidelines (21). The remaining volume of the semen samples was cryopreserved until SCSA analysis was performed. The 200 frozen semen samples from normal control fertile men were collected and donated by the research department of Zhejiang Xingbo Biological Technology Company. All the males included in the control group had their new born babies within the recent two years and their sperm quality were qualified according to the fifth edition of WHO laboratory manual for examination and processing of human semen.

\subsection{SCSA}

SCSA is a sensitive technology based on a flow cytometric technique measuring the susceptibility of sperm DNA to acid which can induce DNA denaturation in situ (21). By using SCSA, we can evaluate sperm DNA fragmentation index (DFI) and detect spermatozoa with abnormal sperm chromatin structure consisting of abnormal nuclear proteins which is described as high DNA stainability (HDS). We performed SCSA by using a commercial kit produced by Zhejiang Xingbo Biological Technology Company according to standard procedure $(20,22,23)$. The sperm count $\left(\times 10^{6} / \mathrm{mL}\right)$ was adjusted with buffer A (comprised of Tris-HCL, $\mathrm{NaCl}$, EDTA) in the kit according to the requirements for SCSA. Briefly, the sperm count was adjusted to $1-2 \times 10^{6} / \mathrm{mL}$ and was prepared in a total of $100 \mathrm{uL}$. Then, $200 \mathrm{uL}$ buffer B (comprised of HCL, $\mathrm{NaCl}$, Triton X-100) was added to the tube. After 30 seconds, sperm cells were stained by adding $600 \mathrm{uL}$ acridine orange (AO) staining (including $\mathrm{Na}_{2} \mathrm{HPO}_{4}$, EDTA, NaCl). After 3 minutes staining, a total of 10,000 sperm cells were analyzed by flow cytometry (FACS Calibur, Becton Dickinson). By staining with the fluorescent dye acridine orange (AO), double stranded native DNA emits green fluorescence, while fragmented single stranded DNA emits red fluorescence. The data was obtained from a specific dedicated software offered by the Xingbo Corporation. The extent of DNA denaturation is indicated by the percentage of sperm containing fragmented DNA after acid treatment, that is, shifting from green fluorescence to red fluorescence after an acidic treatment $(7,18,22,24)$. A scatter plot was created, DFI was expressed as ratio of red to total (red plus green) fluorescence, that is, the ratio of sperm with denatured DNA over total sperm. The percentage of sperm with high DNA stainability (HDS) in each sample was also recorded from the graph plot.

\subsection{Statistical analysis approach}

Statistical analysis was performed using the Statistics Package for the Social Sciences software (SPSS), version 23.0. The classification data was represented by frequency and percentage. Chi-square test was used for group comparisons. The normality test was firstly conducted for the measurement data. If the normal distribution was satisfied, the data was represented as $\mathrm{X}($ Mean $) \pm \mathrm{S}$ (standard deviation). The $t$ test of independent sample data was used for comparison between the two groups. Where the normal distribution was not satisfied, the median and quartile numbers were used to represent it. Kruskal-wallis $H$ test was used for comparison among multiple groups, and Mann-Whitney $U$ test was used for pairwise comparisons. Receiver operating characteristic (ROC) curve analysis was applied to evaluate the ability of DFI to differentiate RSA patients from controls, by calculating the corresponding truncation value, sensitivity and specificity. In this study, a bilateral test was used, and $p<0.05$ was considered statistically significant. The correlation between DFI and semen parameters was determined by using Pearson's correlation coefficient. The level of significance was established at $95 \%$ of the confidence interval in order to be considered statistically significant.

\section{Results}

DFI median and range values as well as its comparison between 139 RSA semen samples and 200 normal controls are shown in Table 1 . The results showed that the DFI median of RSA male partner was $13.84 \%$ (ranged 10.38-23.57\%), which was significantly higher

Table 1. Comparison of sperm DNA fragmentation (DFI) between fertile controls and RSA male partners and distribution percentage of different levels of DFI in both groups

\begin{tabular}{lccr}
\hline Parameter Category & Normal controls $(n=200)$ & RSA male partners $(n=139)$ & Chi-square \\
\hline DFI (\%) & $9.86(8.45-11.11)$ & $13.84(10.38-23.57)^{*}$ & 56.389 \\
$<15 \%$ & $187(93.5 \%)$ & $74(53.2 \%)^{*}$ & 83.354 \\
$15-30 \%$ & $13(6.5 \%)$ & $47(33.8 \%)^{*}$ & 95.635 \\
$>30 \%$ & $0(0.0 \%)$ & $18(12.9 \%)$ & $<0.001$ \\
\hline
\end{tabular}

DFI values are presented as median(range); *compared with normal controls, $p<0.05$ and considered statistically significant. 

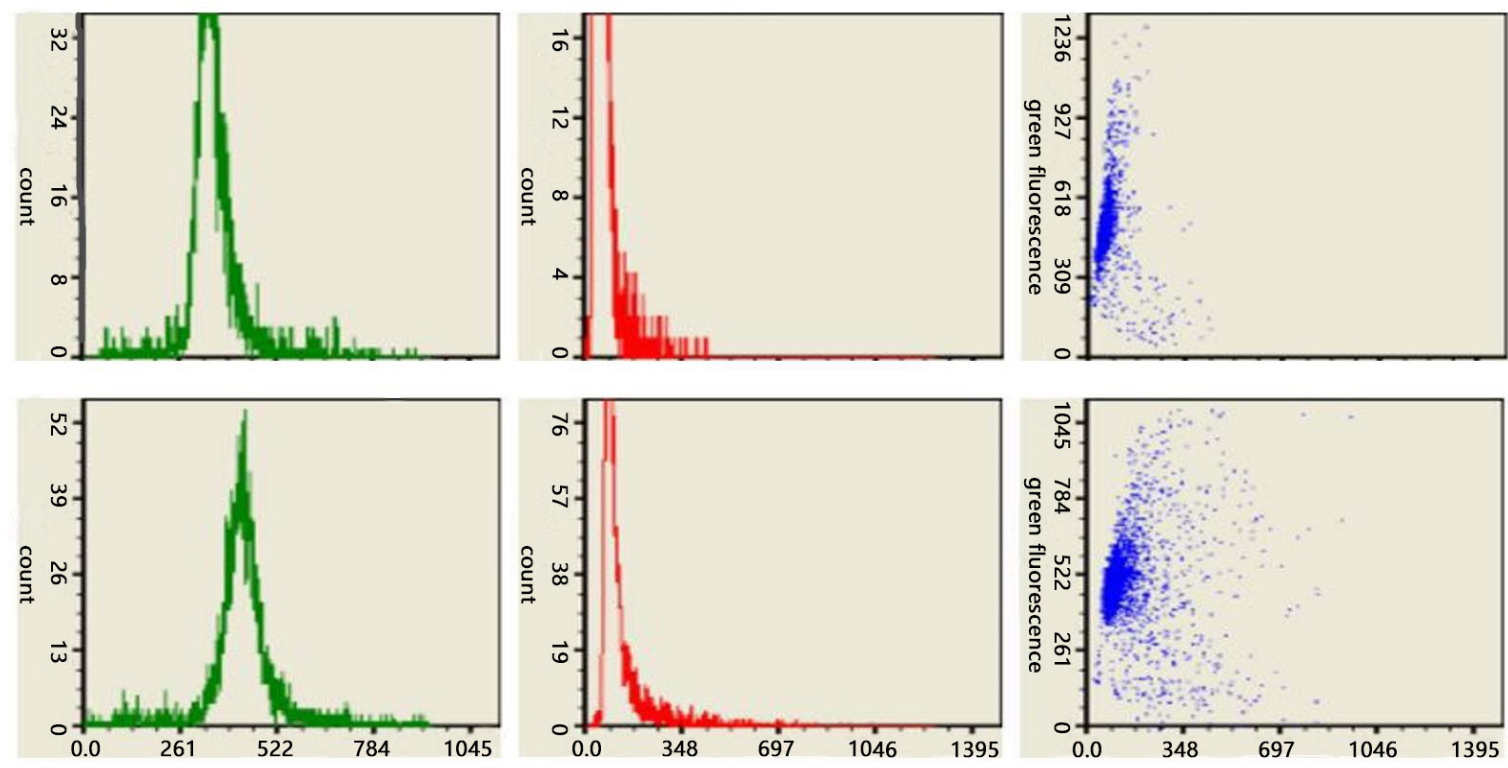

A

B

C

Figure 1. Florescent plots of two semen samples using SCSA. (A, B) Green fluorescence represents sperm with complete DNA, and red fluorescence represents sperm with fragmented DNA. X-axis: fluorescence intensity; Y-axis: sperm count; (C) Scatter diagram of sperm by SCSA, X-axis represents red fluorescence emitted from sperm with fragmented DNA and Y-axis represents green fluorescence emitted from sperm with complete DNA. Upper row: florescent plot of a semen sample from control group with DFI value of 5.7\%; Lower row: florescent plot of a semen sample from RSA patient with DFI value of $17.1 \%$.

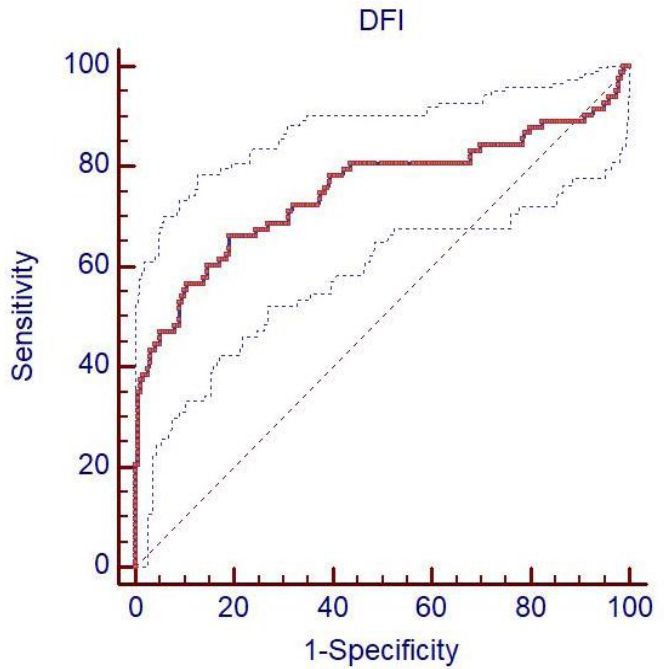

Figure 2. Receiver operating characteristics curve (ROC) analysis of DFI in RSA male patients to normal control group. ROC curve analysis of sperm DFI to diagnose male related RSA. DFI cutoff value: $13.59 \%$; AUC (area under curve): 0.752 ; $95 \%$ CI (confidence interval): 0.697-0.801; sensitivity: $66.27 \%$; specificity: $81.00 \%$.

than that of normal controls, which was $9.86 \%$ (range $8.45-11.11 \%$ ). Analysis of the three DFI level groups showed that the percentage of males with DFI $<15 \%$ was significantly higher $(p<0.001)$ in the control group $(93.5 \%)$ than in RSA groups $(53.2 \%)$. While on the contrary, for the other two DFI level groups (15$30 \%$ and $>30 \%$ respectively), RSA couples showed a significantly higher percentage than the fertile controls.

Figure 1 is a schematic diagram of a sperm fluorescent histogram plot from a normal male and a male patient by using SCSA and software fitting. The DFI was $5.7 \%$ and $17.1 \%$ respectively. Receiver operating characteristics curve (ROC) analysis of DFI in RSA patients compared to normal control group is shown in Figure 2. The DFI cut-off value with highest sensitivity and specificity for diagnosing RSA patients was found at $13.59 \%$. When the threshold level $13.59 \%$ was used in RSA patients, the sensitivity and specificity was estimated as $66.27 \%$ and $81.00 \%$ respectively.

The correlation between DFI tested by SCSA and conventional seminal parameters was also analyzed. The 139 patients were divided into two groups, defined by the DFI threshold level of $13.59 \%(<13.59 \%$ group $v s \geq$ $13.59 \%$ group). Student's $t$-test was applied to compare the two groups, and $p<0.05$ was considered statistically significant. Non-normal distribution data is expressed as median (range). We found a significant difference in abstinence days and progressive motility $(p<0.05)$ but not sperm count, viability or normal morphology rate $(p$ $>0.05$ ) between the two groups (Table 2).

DFI and HDS were compared between different groups in four semen analysis parameters and are shown in Table 3. The parameters included abstinence days, sperm count, viability and progressive motility. Comparison of DFI and HDS levels among three groups classified by abstinence days showed that when comparing $>7$ days of abstinence group with 3-7 days and $<3$ days group, there was a tendency of decrement 
Table 2. Comparison of conventional seminal parameters in RSA husbands with different DNA fragment levels divided by the threshold value $(13.59 \%)$ of sperm DFI

\begin{tabular}{|c|c|c|c|c|}
\hline Semen parameters & Sperm DFI $<13.59 \%$ group, $n=76$ & Sperm DFI $\geq 13.59 \%$ group, $n=63$ & $\mathrm{Z} / \mathrm{t}$ & $p$ \\
\hline Abstinence days & $4.00(3.00-5.00)$ & $5.00(4.00-7.00)$ & -3.012 & $0.003 *$ \\
\hline Sperm count $\left(\times 10^{6} / \mathrm{mL}\right)$ & $52.22(24.45-78.40)$ & $65.05(33.49-97.75)$ & -1.658 & 0.097 \\
\hline Viability $(\%)$ & $48.02 \pm 18.76$ & $42.97 \pm 20.82$ & 1.376 & 0.172 \\
\hline Progressive motility (\%) & $37.56 \pm 14.31$ & $32.01 \pm 15.63$ & 1.997 & $0.048^{*}$ \\
\hline Normal morphology (\%) & $80.00(70.00-80.00)$ & $75.00(65.00-80.00)$ & -1.686 & 0.092 \\
\hline
\end{tabular}

$* p<0.05$ was considered statistically significant.

Table 3. Comparison of DFI and HDS levels of groups classified by different situation of conventional parameters

\begin{tabular}{|c|c|c|c|c|}
\hline Items & DFI (\%) & $p$ value & HDS (\%) & $p$ value \\
\hline \multicolumn{5}{|c|}{ Abstinence days } \\
\hline$<3$ days & $10.36(5.62-13.66)$ & & $7.06(4.58-8.75)$ & \\
\hline 3-7 days & $12.29(8.71-18.54)$ & $0.043 \#$ & $6.29(4.62-8.96)$ & 0.678 \\
\hline$>7$ days & $18.31(12.54-25.99)$ & $0.026^{*}$ & $4.44(3.28-5.23)$ & 0.052 \\
\hline \multicolumn{5}{|c|}{ Sperm count $\left(\times 10^{6} / \mathrm{mL}\right)$} \\
\hline$<15$ & $8.89(7.61-13.29)$ & & $6.41(4.45-9.51)$ & \\
\hline$\geq 15$ & $13.19(8.83-20.51)$ & 0.038 & $5.91(4.51-8.37)$ & 0.618 \\
\hline \multicolumn{5}{|l|}{ Viability (\%) } \\
\hline$<58$ & $10.38(8.89-19.20)$ & & $6.30(4.60-8.43)$ & \\
\hline$\geq 58$ & $9.36(8.30-17.43)$ & 0.162 & $5.63(3.88-8.28)$ & 0.216 \\
\hline \multicolumn{5}{|c|}{ Progressive motility (\%) } \\
\hline$<32$ & $15.41(10.55-21.72)$ & & $6.41(4.95-9.81)$ & \\
\hline$\geq 32$ & $10.56(8.30-15.76)$ & 0.002 & $5.63(4.36-7.59)$ & 0.085 \\
\hline
\end{tabular}

$p<0.05$ was considered significant; $*>7$ days group compared with $<3$ days group, $p<0.05$; \#> 7 days group compared with 3-7 days group, $p<0.05$.

in DFI and the differences were significant $(p<0.05)$, while comparison of HDS showed no significance (Table 3). To our surprise, sperm count $<15 \times 10^{6}$ group showed a significant lower median DFI level compared with $\geq 15 \times 10^{6}$ group, which was $8.89 \%$ (range $7.61 \%$ $13.29 \%$ ) and $13.19 \%$ (range $8.83 \%-20.51 \%$ ) respectively $(p<0.05)$, while there was no significant difference of HDS level between the two groups. We analyzed the possible relationship between sperm DNA fragmentation and conventional sperm parameters of sperm viability and progressive motility. Neither DFI nor HDS levels showed significant difference $(p>0.05)$ between two groups classified by $5^{\text {th }}$ WHO standard of $58 \%$ for sperm viability (\%) by using SCSA. As for the comparison of DFI and HDS levels between two groups classified by sperm progressive motility rate $[\mathrm{PR}(\mathrm{A}+\mathrm{B}) \%]$ of $32 \%$, the result showed a significant $(p<0.05)$ positive relationship between sperm DNA integrity and sperm progressive motility rate. However the result showed no significant difference $(p>0.05)$ in HDS level between the two groups.

Correlation between DFI and different WHO semen parameters was analyzed and calculated, as shown in Table 4. According to our results, abstinence days significantly positively correlated with DFI while sperm viability, progressive motility and normal morphology rate significantly negatively correlated with DFI ( $p$ $<0.05)$. There appeared to be no significant relation between DFI and sperm counts.
Table 4. Correlation analysis between semen parameters and DFI in 139 RSA group samples

\begin{tabular}{lcl}
\hline Items & $r$ & $p$ \\
\hline Abstinence days & 0.332 & $0.000^{*}$ \\
Sperm count $\left(\times 10^{6} / \mathrm{mL}\right)$ & 0.157 & 0.092 \\
Viability $(\%)$ & -0.194 & $0.037^{*}$ \\
Progressive motility (\%) & -0.266 & $0.004^{*}$ \\
Normal morphology $(\%)$ & -0.248 & $0.009^{*}$ \\
\hline
\end{tabular}

" $p<0.05$ was considered statistically significant; $r=$ correlation coefficient.

\section{Discussion}

The etiology of reduced semen quality remains unexplained. Sperm DNA or chromatin damage has been reported as mainly linked to abnormal or incomplete chromatin packaging during spermatogenesis, abortive apoptosis and oxidative stress induced by releasing of reactive oxygen species (ROS) $(25,26)$. The oxidative damage is associated with exposure to a variety of genotoxic risk agents including environmental exposure, diseases, life styles, and et al. $(1,27,28)$. Sperm that lacks antioxidants and DNA repair systems, as well as DNA repair from oocytes or early embryo are vulnerable to DNA strand damage. Although their correlation was not always found statistically significant, numerous studies tended to believe that a high level of SDF is a deleterious factor 
for achieving and sustaining term pregnancies (1).

A significant negative correlation between sperm DNA damage and embryo quality has been elucidated in vitro and in vivo (29) and high DFI has proven to be associated with increased miscarriage rate $(19,30)$. The high degree of DNA fragmentation may not necessarily affect fertilization rates, but may result in subsequent miscarriage. From the present work we perceive that patients diagnosed with male caused RSA have a significantly higher level of DFI compared to normal controls (Table 1). Thus, our study further supports the value of SDF testing used in clinical investigation for male caused RSA.

According to the former report, when DFI is higher than $20 \%$, the chance of obtaining a natural pregnancy is decreased, and close to zero when DFI is over 30$40 \%$ (31). It's now widely accepted that sperm with DFI $>15 \%$ (percentage offered by most commercial kits) is not ideal and patients with DFI $>30 \%$ are at higher risk of infertility regardless of whether they conceive via natural conception or ART. However, there is still no specific diagnostic threshold for specific infertility circumstances such as RSA. Besides, different ethnic population and different reagents or instruments used in different laboratories may be factors affecting the diagnostic accuracy of DFI results. Therefore, it is necessary for the laboratory to establish its own diagnostic threshold. According to our present work, we defined our own cutoff value as $13.59 \%$ using SCSA and its sensitivity and specificity was evaluated as $66.27 \%$ and $81.00 \%$, respectively (Figure 1). Both the sensitivity and specificity of the DFI threshold were not very ideal, indicating that DFI is only one of the determinants which are important for the underlying causes of RSA.

It was shown that $25-40 \%$ of infertile men may have normal sperm characteristics according to WHO criteria, but with DFI $>20-30 \%(32,33)$. Thus, controversies regarding the correlations between DFI and conventional semen parameters exist. Several studies reported a weak-to-moderate inverse correlation between DFI and sperm count, motility, vitality and morphology $(19,34)$. Our correlation study of 139 RSA cases showed a positive correlation between DFI level and abstinence days. This may be related to various adverse factors such as ROS on sperm DNA integrity during sperm's transmission to the epididymis. Our results also revealed an only weak negative correlation between DFI and viability, motility and normal morphological rate (Table 4). Since 'normal' sperm defined by conventional semen analysis may still carry fragmented DNA, we propose that sperm DNA integrity should be included in sperm quality tests and used as a parameter independent of conventional semen analysis.

HDS provides information on chromatin condensation associated with sperm cell immaturity. High HDS rate have negative effects on pregnancy and has been proven to be an effective indicator of possible spontaneous abortion in assisted reproduction, and the correlation between HDS $>15 \%$ and low fertilization rates in IVF was found $(19,31)$. However, in our study, we found no significant difference of HDS between groups classified by routine semen parameters. In the future research, it is expected to enlarge the study sample size and further explore the relationship between HDS and fertility.

In summary, we conclude that DFI level of RSA males is significantly higher than that of normal controls which is accordance with some previous reports $(2,7,35)$. We strongly recommend the use of SDF testing besides routine semen analysis to assess a male's fertility more comprehensively. We established a DFI threshold value of $13.59 \%$ for our own laboratory, which should be helpful for the establishment of a reference value of Chinese RSA patients. According to our work, lowering the DFI values to below the threshold of $13.59 \%$ through lifestyle management or medication is expected to increase the success rate of pregnancy in patients with repeated abortions. More investigations on the relation between DFI or HDS levels and pregnancy outcomes on a larger sample size are still waiting to be carried out. Understanding the precise mechanism of sperm DNA damage as well as exploring the corresponding clinical interventions that give the best chances of full term pregnancy are also of great importance.

\section{Acknowledgements}

This work was supported by grants from the National Natural Science Foundation of China (no. 31571196 to Ling Wang and no. 30801502 to Ling Wang), the Program to Guide Medicine ("Yixueyindao") of the Shanghai Municipal Science and Technology Commission (no. 18401902200 to Ling Wang and no. 15401932200 to Ling Wang), the Shanghai Program for Support of Leading Disciplines-Integrative Medicine (no. 20180101 and no. 20150407), the Research Foundation ("CR Sanjiu") of Obstetrics \& Gynecology committee of Chinese Association of Integrated Traditional Chinese and Western Medicine (CR1901FC01 to Ling Wang), the Shanghai Committee of the China Democratic League (no. 02054 to Ling Wang), the FY2008 JSPS Postdoctoral Fellowship for Foreign Researchers (P08471 to Ling Wang), and the Shanghai Pujiang Program (no. 11PJ1401900 to Ling Wang). We also appreciate Huijing Tang and Jing Liu, laboratory technicians from the Department of Clinical Testing, for their support and assistance in data collection and analyzing the traditional semen parameters.

\section{References}

1. Evgeni E, Charalabopoulos K, Asimakopoulos B. Human 
sperm DNA fragmentation and its correlation with conventional semen parameters. J Reprod Infertil. 2014; 15:2-14.

2. Lin MH, Kuo-Kuang Lee R, Li SH, Lu CH, Sun FJ, Hwu YM. Sperm chromatin structure assay parameters are not related to fertilization rates, embryo quality, and pregnancy rates in in vitro fertilization and intracytoplasmic sperm injection, but might be related to spontaneous abortion rates. Fertil Steril. 2008; 90:352359.

3. van der Steeg JW, Steures P, Eijkemans MJ, JD FH, Hompes PG, Kremer JA, van der Leeuw-Harmsen L, Bossuyt PM, Repping S, Silber SJ, Mol BW, van der Veen F, Collaborative Effort for Clinical Evaluation in Reproductive Medicine Study G. Role of semen analysis in subfertile couples. Fertil Steril. 2011; 95:1013-1019.

4. Ahmadi A, Ng SC. Developmental capacity of damaged spermatozoa. Hum Reprod. 1999; 14:2279-2285.

5. Carrell DT, Liu L, Peterson CM, Jones KP, Hatasaka HH, Erickson L, Campbell B. Sperm DNA fragmentation is increased in couples with unexplained recurrent pregnancy loss. Arch Androl. 2003; 49:49-55.

6. Ford HB, Schust DJ. Recurrent pregnancy loss: Etiology, diagnosis, and therapy. Rev Obstet Gynecol. 2009; 2:7683.

7. Absalan F, Ghannadi A, Kazerooni M, Parifar R, Jamalzadeh F, Amiri S. Value of sperm chromatin dispersion test in couples with unexplained recurrent abortion. J Assist Reprod Genet. 2012; 29:11-14.

8. Khadem N, Poorhoseyni A, Jalali M, Akbary A, Heydari ST. Sperm DNA fragmentation in couples with unexplained recurrent spontaneous abortions. Andrologia. 2014; 46:126-130.

9. Coughlan C, Clarke H, Cutting R, Saxton J, Waite S, Ledger W, Li T, Pacey AA. Sperm DNA fragmentation, recurrent implantation failure and recurrent miscarriage. Asian J Androl. 2015; 17:681-685.

10. Bellver J, Meseguer M, Muriel L, Garcia-Herrero S, Barreto MA, Garda AL, Remohi J, Pellicer A, Garrido N. Y chromosome microdeletions, sperm DNA fragmentation and sperm oxidative stress as causes of recurrent spontaneous abortion of unknown etiology. Hum Reprod. 2010; 25:1713-1721.

11. Basar MM, Kahraman S. Clinical utility of sperm DNA fragmentation testing: Practice recommendations based on clinical scenarios. Transl Androl Urol. 2017; 6:S574-S576.

12. Spanò M, Bonde JP, Hjøllund HI, Kolstad HA, Cordelli E, Leter G. Sperm chromatin damage impairs human fertility. The Danish First Pregnancy Planner Study Team. Fertil Steril. 2000; 73:43-50.

13. Ribas-Maynou J, Garcia-Peiro A, Fernandez-Encinas A, Amengual MJ, Prada E, Cortes P, Navarro J, Benet J. Double stranded sperm DNA breaks, measured by Comet assay, are associated with unexplained recurrent miscarriage in couples without a female factor. PLoS One. 2012; 7:e44679.

14. Tamburrino L, Marchiani S, Montoya M, Elia Marino F, Natali I, Cambi M, Forti G, Baldi E, Muratori M. Mechanisms and clinical correlates of sperm DNA damage. Asian J Androl. 2012; 14:24-31.

15. Bungum M, Bungum L, Giwercman A. Sperm chromatin structure assay (SCSA): A tool in diagnosis and treatment of infertility. Asian J Androl. 2011; 13:69-75.

16. Cho CL, Agarwal A, Majzoub A, Esteves SC. Clinical utility of sperm DNA fragmentation testing: Concise practice recommendations. Transl Androl Urol. 2017; 6:S366-S373

17. Chi HJ, Chung DY, Choi SY, Kim JH, Kim GY, Lee JS, Lee HS, Kim MH, Roh SI. Integrity of human sperm DNA assessed by the neutral comet assay and its relationship to semen parameters and clinical outcomes for the IVF-ET program. Clin Exp Reprod Med. 2011; 38:10-17.

18. Evenson DP, Darzynkiewicz Z, Melamed MR. Relation of mammalian sperm chromatin heterogeneity to fertility. Science. 1980; 210:1131-1133.

19. Boe-Hansen GB, Fedder J, Ersbøll AK, Christensen P. The sperm chromatin structure assay as a diagnostic tool in the human fertility clinic. Human Reproduction. 2006; 21:1576-1582.

20. Evenson DP, Jost LK, Marshall D, Zinaman MJ, Clegg E, Purvis K, de Angelis P, Claussen OP. Utility of the sperm chromatin structure assay as a diagnostic and prognostic tool in the human fertility clinic. Human Reproduction. 1999; 14:1039-1049.

21. World Health Organization. WHO laboratory manual for the examination and processing of human semen. 5th ed. Geneva : World Health Organization, 2010; pp. 10-44.

22. Evenson DP, Larson KL, Jost LK. Sperm chromatin structure assay: its clinical use for detecting sperm DNA fragmentation in male infertility and comparisons with other techniques. J Androl. 2002; 23:25-43.

23. Jurewicz J, Radwan M, Wielgomas B, Sobala W, Piskunowicz M, Radwan P, Bochenek M, Hanke W. The effect of environmental exposure to pyrethroids and DNA damage in human sperm. Syst Biol Reprod Med. 2015; 61:37-43.

24. Imam SN, Shamsi MB, Kumar K, Deka D, Dada R. Idiopathic recurrent pregnancy loss: Role of paternal factors; a pilot study. J Reprod Infertil. 2011; 12:267-276.

25. Moustafa MH, Sharma RK, Thornton J, Mascha E, Abdel-Hafez MA, Thomas AJ, Jr., Agarwal A. Relationship between ROS production, apoptosis and DNA denaturation in spermatozoa from patients examined for infertility. Hum Reprod. 2004; 19:129-138.

26. Talebi AR, Fesahat F, Mangoli E, Ghasemzadeh J, Nayeri M, Sadeghian-Nodoshan F. Relationship between sperm protamine deficiency and apoptosis in couples with unexplained repeated spontaneous abortions. Int J Reprod Biomed (Yazd). 2016; 14:199-204.

27. Aitken RJ, De Iuliis GN. On the possible origins of DNA damage in human spermatozoa. Mol Hum Reprod. 2010; 16:3-13

28. Sakkas D, Alvarez JG. Sperm DNA fragmentation: Mechanisms of origin, impact on reproductive outcome, and analysis. Fertil Steril. 2010; 93:1027-1036.

29. Erenpreiss J, Spano M, Erenpreisa J, Bungum M, Giwercman A. Sperm chromatin structure and male fertility: Biological and clinical aspects. Asian J Androl. 2006; 8:11-29.

30. Zhao J, Zhang Q, Wang Y, Li Y. Whether sperm deoxyribonucleic acid fragmentation has an effect on pregnancy and miscarriage after in vitro fertilization/ intracytoplasmic sperm injection: A systematic review and meta-analysis. Fertil Steril. 2014; 102:998-1005 e1008.

31. Kennedy C, Ahlering P, Rodriguez H, Levy S, Sutovsky P. Sperm chromatin structure correlates with spontaneous abortion and multiple pregnancy rates in assisted 
reproduction. Reprod Biomed Online. 2011; 22:272-276.

32. Erenpreiss J, Elzanaty S, Giwercman A. Sperm DNA damage in men from infertile couples. Asian J Androl. 2008; 10:786-790.

33. Zini A, Sigman M. Are tests of sperm DNA damage clinically useful? Pros and cons. J Androl. 2009; 30:219229.

34. Giwercman A, Richthoff J, Hjøllund H, Bonde JP, Jepson K, Frohm B, Spano M. Correlation between sperm motility and sperm chromatin structure assay parameters. Fertil Steril. 2003; 80:1404-1412.

35. Brahem S, Mehdi M, Landolsi H, Mougou S, Elghezal H, Saad A. Semen Parameters and Sperm DNA Fragmentation as Causes of Recurrent Pregnancy Loss. Urology. 2011; 78:792-796.

(Received December 7, 2018; Revised March 16, 2019; Accepted March 26, 2019) 